Nota Técnica

Pensar en Movimiento:

Revista de Ciencias del Ejercicio y la Salud

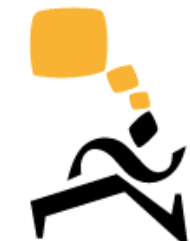

PENSAR EN

EISSN: $1659-4436$

Vol. 13, No. 1, pp. 1- 10

Cierre al 30 de junio, 2015

\title{
CAPACIDAD DEL MÉTODO DE PARCHES DE SUDORACIÓN PARA DETECTAR EL EFECTO DE UN ANTITRANSPIRANTE
}

\section{CAPACITY OF A SWEAT PATCH METHOD TO DETECT THE EFFECT OF AN ANTIPERSPIRANT}

\section{CAPACIDADE DO MÉTODO DE ADESIVOS DE SUOR PARA DETECTAR O EFEITO DUM ANTITRANSPIRANTE}

\author{
Jorge Alberto Aburto Corona, Lic ${ }^{1(B, C, D, E)}$ y Luis Fernando \\ Aragón Vargas, Ph.D.; FACSM ${ }^{2(B, D, E)}$ \\ jorge.alberto.aburto@gmail.com \\ 'Estudiante del programa de Maestría en Ciencias del Movimiento Humano, Escuela de \\ Educación Física y Deportes, Universidad de Costa Rica, Costa Rica \\ ${ }^{2}$ Centro de Investigación en Ciencias del Movimiento Humano y Escuela de \\ Educación Física y Deportes, Universidad de Costa Rica, Costa Rica
}

Fecha de recepción: 28-07-2014 Fecha de reenvíos: 26-11-2014

Fecha de aceptación: 09-01-2015 Fecha de publicación: 18-03-2015

doi: http://dx.doi.org/10.15517/pensarmov.v13i1.15404

\section{Resumen}

Aburto-Corona, J.A. y Aragón-Vargas, L.F. (2015). Nota técnica: Capacidad del método de parches de sudoración para detectar el efecto de un antitraspirante. PENSAR EN MOVIMIENTO: Revista de Ciencias del Ejercicio y la Salud, 13 (1), 1-10. Un antitranspirante efectivo es supuestamente capaz de disminuir la sudoración hasta en un $50 \%$, como lo afirman las compañías que fabrican estos productos, pero hay poca evidencia publicada al respecto; es posible que la disminución real sea tan pequeña que no pueda ser detectada por los

$$
-1 \text { - }
$$


métodos convencionales. El propósito de este estudio fue verificar si el método de parches de sudoración es capaz de mostrar una disminución en la tasa de sudoración localizada que debería ocurrir por el uso de un antitranspirante. Además, se midió la magnitud de este efecto. La influencia esperada del antitranspirante (condición $A$, piel rociada con antitranspirante) se verificó con base en la tasa de sudoración localizada de la zona escapular durante 20 minutos de ejercicio a una intensidad entre 78 y $80 \%$ de la FCmáx con una temperatura ambiental promedio de $29,7 \pm 0,5^{\circ} \mathrm{C}$ y una humedad relativa de $54 \pm 3,4 \%$, comparada con una condición control (C) (piel sin antitranspirante). Por consiguiente, se encontraron diferencias estadísticamente significativas entre las condiciones $A=14,6 \pm 10,3$ $\mu \mathrm{L} * \min ^{-1}$ y $\mathrm{C}=19,2 \pm 12,6 \mu \mathrm{L} * \min ^{-1}(\mathrm{p}=0,001)$ en la tasa de sudoración de la zona escapular, lo cual significa que los participantes sudaron aproximadamente un $24 \%$ menos cuando se les aplicó antitranspirante comparado con la piel intacta, según las mediciones obtenidas con el método de parches de sudoración.

Palabras clave: Cloruro de aluminio; láminas de sudor; loción corporal; termorregulación.

\begin{abstract}
Aburto-Corona, J.A. y Aragón-Vargas, L.F. (2015). Nota técnica: Capacity of a sweat patch method to detect the effect of an antiperspirant. PenSAR en Movimiento: Revista de Ciencias del Ejercicio y la Salud, 13 (1), 1-10. An effective antiperspirant is supposed to reduce sweat production by up to $50 \%$, according to the manufacturers, but the scientific evidence in the literature is limited; possibly, the actual reduction may be so small that it may not be detected by conventional methods. The purpose of this study was to verify if the sweat patch method was able to detect the reduction in regional sweat loss that would be expected from using an antiperspirant. In addition, the magnitude of the effect was quantified. We verified the impact of an antiperspirant (condition $A$, skin treated with the product) on localized scapular sweat rate during 20 minutes of exercise at $78-80 \%$ HRmax, at $29.7 \pm$ $0.5^{\circ} \mathrm{C}$ of ambient temperature and $54 \pm 3.4 \%$ relative humidity, compared with a control condition ( $\mathrm{C}$, untreated skin). A statistically significant difference in scapular sweat rate was found between conditions: $A=14.6 \pm 10.3 \mu \mathrm{L} * \min ^{-1}$ and $C=19.2 \pm 12.6 \mu \mathrm{L} * \mathrm{~min}^{-1}(p=$ $0.001)$. This means that the participants secreted approximately $24 \%$ less sweat when using an antiperspirant, compared with the intact skin, as measured by the sweat patch method.
\end{abstract}

Key words: Aluminum chloride; sweat sheets; body lotion; thermoregulation.

\title{
Resumo
}

Aburto-Corona, J.A. y Aragón-Vargas, L.F. (2015). Nota técnica: Capacidade do método de adesivos de suor para detectar o efeito dum antitranspirante. PENSAR EN Movimiento: Revista de Ciencias del Ejercicio y la Salud, 13 (1), 1-10. Supõe-se que um -2 -

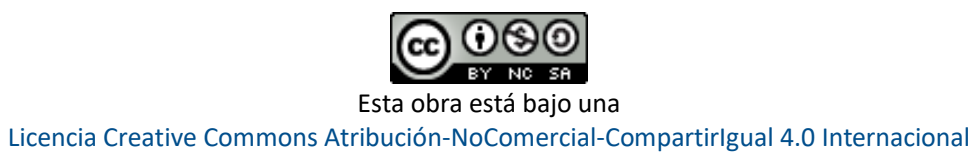


antitranspirante efetivo é capaz de diminuir o suor até $50 \%$ como afirmam as companhias que fabricam isto produtos, mas são poucas evidências a respeito do mesmo: é possível que a real diminuição seja pequena não podendo ser detectada com métodos convencionais. $O$ objetivo deste estudo foi verificar se o método de adesivos de suor é capaz de detectar a diminuição da taxa de transpiração localizada que deve ocorrer a través da utilização de um antitranspirante. Alem disso, a magnitude do efeito foi medido. Verificou-se a influencia que tem o antitranspirante (Condição $A$, pele rociada com antitranspirante) na taça de sudoração localizada da zona escapular durante 20 minutos de exercícios na intensidade entre $78 \%$ e $80 \%$ da FCmax com temperatura ambiental media de $29.7 \pm 0.5^{\circ} \mathrm{C}$ e humidade relativa de 54 $\pm 3.4 \%$, comparado com uma condição controle (C) (pele sim antitranspirante). Controlaramse diferenças estatisticamente significativas entre as condições $A=14.6 \pm 10.3 \mu \mathrm{L}$ * $\min -1 \mathrm{y}$ $C=19.2 \pm 12.6 \mu \mathrm{L} * \min -1$ ( $p=0.001)$ na taça de sudoração da zona escapular, ou seja, que os participantes suaram aproximadamente $24 \%$ menos quando se aplico antitranspirante comparado com a pele intata, de acordo com as medições obtidas com as adisivos de suor.

Palavras chave: Cloreto de alumínio; manchas de suor; loção para o corpo; termorregulação.

La termorregulación durante la actividad física se ha estudiado en detalle desde hace aproximadamente cien años (Burton, 1934). Desde entonces se ha realizado un número limitado de estudios sobre la tasa de sudoración localizada (TSL) (Bain, Deren \& Jay, 2011). Un método utilizado para recolectar el sudor de determinada zona corporal son los parches de sudor, los cuales eran empleados originalmente por las autoridades para la detección de drogas en el organismo (Phillips \& McAllon, 2008), su uso fue tan eficaz para la recolección de sudor que se volvió un material indispensable en algunas áreas de termorregulación, particularmente en estudios relacionados con el deporte (Havenith, Fogarty, Bartlett, Smith \& Ventenat, 2008; Morris, Cramer, Hodder, Havenith \& Jay, 2013; Shirreffs, Aragón-Vargas, Chamorro, Maughan, Serratosa \& Zachwieja, 2005).

Por lo tanto, sería de mucha utilidad verificar si el método de parches de sudoración, modificado de Maughan, Merson, Broad \& Shirreffs (2004), es suficientemente sensible para la detección de diferencias pequeñas en la tasa de sudoración localizada obtenidas en condiciones de ejercicio moderado. Una forma de inducir estas diferencias sería aplicando un antitranspirante, pues se esperaría que dicha sustancia disminuyera la tasa de sudoración. Cabe mencionar que este método (de parches) es comúnmente utilizado en condiciones de ejercicio intenso en calor y sudoración profusa para medir concentraciones de electrolitos, no para comparar la tasa de sudoración.

Algunas investigaciones previas han usado antitranspirante en personas con hiperhidrosis (sudor excesivo) (Reynolds, et al., 1995; Schmidt-Rose, et al., 2013). En estos estudios, a los sujetos solo se les evalúo la tasa de sudoración en manos, pies y axilas, 
mientras fueron sometidos a situaciones de estrés psicosocial o con ejercicio de muy baja intensidad como caminar. En los estudios de estrés psicosocial (en los cuales se emplearon parches de sudor) se ha encontrado que los tres tipos de presentaciones de antitranspirantes (roll-on, aerosol y en barra) son efectivos para disminuir la sudoración localizada, cuando los sujetos son sometidos a situaciones de estrés social mediante una prueba mental (SchmidtRose, et al., 2013), en cambio, en los estudios de ejercicio a baja intensidad (en donde se pesa la prenda antes y después de la prueba) no hallaron diferencias estadísticamente significativas entre piel rociada con antitranspirante y piel sin antitranspirante cuando los sujetos caminaban en una banda sin fin $(1,39 \mathrm{~m} / \mathrm{seg}, 1 \%$ inclinación) en un ambiente caliente $\left(28^{\circ} \mathrm{C}, 25 \%\right.$ de humedad relativa) durante 200 minutos (Reynolds, et al., 1995).

También, existe otro estudio en condiciones de ejercicio de alta intensidad en el cual los investigadores no encontraron diferencias significativas en la tasa de sudoración con o sin uso de antitranspirante (Burry, Evans, Rawlings \& Shiu, 2003). Estos estudios presentan varias limitaciones metodológicas que podrían haber incidido en los resultados, como la falta de control en la intensidad del ejercicio, en el estado de hidratación, entre otras.

Debido a la necesidad de verificar la capacidad del método de parches para detectar pequeños cambios en la sudoración regional como consecuencia del uso de lociones corporales, y según la lógica de que la aplicación de antitranspirante a la piel sí debería disminuir la tasa de sudoración en esa zona, se diseñó el presente experimento para verificar si este método es capaz de detectar la disminución esperada en la tasa de sudoración cuando se aplica un antitranspirante en la piel y se realiza ejercicio a intensidad moderadaalta en el calor durante 20 minutos. Este método puede ser de gran utilidad para los fabricantes de antitranspirantes y lociones ampliamente utilizadas en el mundo del deporte, pero, también permitiría la evaluación del impacto al utilizar distintas vestimentas o implementos deportivos sobre la termorregulación.

\section{METODOLOGÍA}

Participantes. Para este estudio se reclutaron de manera voluntaria 10 estudiantes universitarios (7 hombres y 3 mujeres), físicamente activos, aparentemente sanos y sin problemas en la piel. A los participantes se les dio indicaciones de que un día antes no debían ingerir medicamentos o alimentos considerados como diuréticos, asimismo, debían ingerir por lo menos 1 litro de agua un día previo a la sesión, con la intención de que la muestra fuera lo más homogénea posible en aspectos de hidratación. Cada participante llevó ropa deportiva (las mujeres usaron top cruzado para mantener descubierta la región escapular; los varones llevaron el dorso desnudo).

Procedimiento. La investigación se realizó en una sola sesión para cada participante, la cual incluyó ambas zonas escapulares (izquierda y derecha) para la condición antitranspirante (A) y la condición control (C) (piel cubierta con loción y piel sin loción, respectivamente). La asignación del tratamiento a cada región escapular se realizó de 
manera aleatoria. Al iniciar la sesión, a cada participante se le entregó un formulario de consentimiento informado y un cuestionario de aptitud para la actividad física "Par-Q" (Adams, 1999), el cual llenó y firmó voluntariamente. También, se le solicitó una muestra de orina para determinar la gravedad específica (GEO) con un refractómetro manual (URC/ $\mathrm{Ne}$, ATAGO ${ }^{\circledR}$, Tokio, Japón), según el método descrito por Aragón-Vargas, Moncada-Jiménez, Hernández, Barrenechea y Monge-Alvarado (2009).

Para la hidratación se utilizó como parámetro el resultado del estudio de Oppliger y Bartok (2002): si su GEO era mayor o igual a 1,020 se encontraba en estado de hipohidratación y no se le permitió participar en la sesión. Se tomó el peso corporal de cada participante, totalmente desnudo, en una báscula calibrada (e-Accura®, modelo DSB291, Romanas Ballar, Costa Rica) y se registró hasta los $10 \mathrm{~g}$ más cercanos (también se le pesó al finalizar la sesión). Seguido, se limpiaron ambas zonas escapulares con gasa y agua destilada (se pasó la gasa remojada 3 veces y con una gasa seca se removió el exceso de agua, solo ejerciendo presión, sin frotar). Se seleccionó la región escapular, ya que es un área donde usualmente no hay vellosidad, es una de las de mayor sudoración y además, según Havenith, Fogarty, Bartlett, Smith y Ventenat (2008), y Smith y Havenith (2011), es una región que suda lo mismo en comparación con la zona contralateral y permitía utilizar ambos lados de esa región. Para seleccionar el punto exacto para la colocación de los parches, se tomó como referencia el manual de las normas internacionales para la evaluación antropométrica (International Society for the Advancement of Kinanthropometry., 2001, p. 32) de la Sociedad Internacional para el Avance de la Cineantropometría (ISAK por sus siglas en inglés): se identificó el borde superior paralelo del punto escapular izquierdo que se menciona en el manual y luego el derecho de la misma manera (ver figura 1).

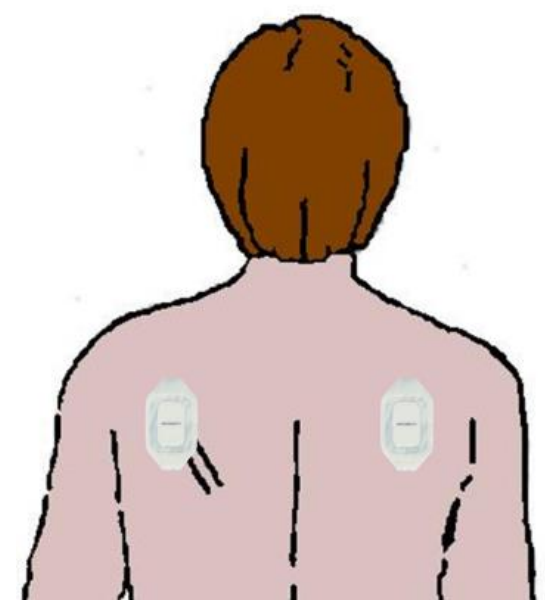

Figura 1. Lugar donde se colocaron los parches de sudor. Para una descripción más detallada, ver el manual de la ISAK (International Society for the Advancement of Kinanthropometry, 2001, p. 32).

Fuente: elaboración propia a partir de http://www.ugr.es/ jhuertas/EvaluacionFisiologica/Antropometria/antroppliegues.htm 
Una vez identificados los puntos escapulares, se procedió a marcarlos con delineador a prueba de agua (Revlon ${ }^{\circledR}$ ColorStyle, Ciudad de Nueva York, Nueva York, Estados Unidos), con ayuda de unos estarcidos diseñados para aplicar la loción de manera exacta en las regiones, de modo que no interfiriera con la parte adherente de los parches. Una vez limpias y marcadas las zonas se aplicó, con ayuda del estarcido, una loción antitranspirante con cloruro de aluminio (Rexona ${ }^{\mathrm{TM}}$ V8 Tuning, Australia) y se dejó secar por 60 minutos (tiempo óptimo que fue determinado en una prueba piloto) mientras los sujetos se mantenían en reposo fuera del cuarto de clima controlado en condiciones ambientales de $28,2 \pm 0,9{ }^{\circ} \mathrm{C}$ de temperatura y 55,1 $\pm 2,2 \%$ de humedad relativa. Pasados los 60 minutos, con una gasa seca se removió el exceso de antitranspirante y se colocó el parche de recolección de sudor de $4,9 \times 3,3 \mathrm{~cm}=16,17 \mathrm{~cm}^{2}$ (MSX - 6446, 3M, Brookings, S.D., Estados Unidos). Para el lado al que le correspondía la condición control no se aplicó nada, solo se colocó el parche en el área marcada cuando se puso el otro en la zona opuesta. Al poner cada parche se puso a correr un cronómetro distinto, con el fin de obtener el tiempo exacto que estuvo el parche en la piel (t). Seguido, los sujetos se ejercitaron en bicicletas estacionarias dentro de un cuarto de clima controlado (temperatura ambiental $=29,7 \pm 0.5^{\circ} \mathrm{C}$, humedad relativa $=54$ $\pm 3,4 \%$ ) durante 20 minutos, a una intensidad moderada-alta entre 78 y $80 \%$ de la frecuencia cardíaca máxima, la cual se calculó conforme a la ecuación: FCmax $=(207-0,7$ * edad) propuesta por Gellis, Goslin, Olson, McDonald, Russi y Moudgil (2007). La intensidad fue verificada con monitores de frecuencia cardíaca (Polar ${ }^{\circledR}$ FT4, Kempele, Finlandia). El peso de los parches se midió con una balanza granataria (A\&D GX - 200, Toshima-ku, Tokio, Japón), antes e inmediatamente después de ser removidos (transcurridos los 20 minutos). Para este procedimiento, se modificó el método de recolección de sudor utilizado por Maughan et al. (2004), y Smith y Havenith (2011). El método consiste en:

1.- Colocar los parches dentro de una bolsa plástica pequeña con cierre hermético (y así impedía que el sudor se evapore) para su pesaje antes y después de terminada la prueba.

2.- Una vez introducidos los parches en la bolsa, esta se dobla (con mucho cuidado para no doblar el parche) y se le coloca un clip de metal, el cual ayuda a que la bolsa de plástico no se expanda y esté variando el peso.

3.- Al tener ambos resultados (peso del parche antes y después de la prueba), se resta y se obtiene un valor. Ese valor (ganancia de peso en gramos) representa la cantidad de sudor absorbido por el parche en un tiempo estipulado, en donde se considera que un gramo de agua equivale a un mililitro, se tomó en cuenta el valor como un volumen de sudor. Este volumen fue estandarizado, se dividió por el tiempo transcurrido entre los dos pesajes y se multiplicó por 1000 para reportar $\mu \mathrm{L}^{*} \mathrm{~min}^{-1}$.

La diferencia de peso en microgramos, el tiempo en minutos y segundos que estuvo colocado cada parche (t) se anotó y a partir de ahí se calculó la tasa de sudoración $=\Delta$ peso $\cdot \mathrm{t}^{-1}$.

Análisis estadístico. Para caracterizar la muestra se realizó estadística descriptiva para la edad, peso corporal (antes y después), talla, $t$, gravedad específica de la orina (GEO) y 
frecuencia cardíaca máxima de los sujetos (ver tabla 1). Se realizó un ANOVA de una vía para muestras relacionadas, con la condición (A o C) como variable independiente y la tasa de sudoración como variable dependiente.

\section{RESULTADOS}

Tabla 1.

Características de los sujetos

\begin{tabular}{cccc}
\hline & Hombres $(\boldsymbol{n}=\mathbf{7})$ & Mujeres $(\boldsymbol{n}=\mathbf{3})$ & Total $(\boldsymbol{n}=\mathbf{1 0})$ \\
\hline Edad (años) & $21,6 \pm 3,1$ & $18,7 \pm 1,2$ & $20,7 \pm 2,9$ \\
Talla (cm) & $176,0 \pm 9,3$ & $159,2 \pm 2,0$ & $170,9 \pm 11,1$ \\
Peso pre (kg) & $74,90 \pm 13,90$ & $51,40 \pm 1,40$ & $69,34 \pm 15,41$ \\
Peso post (kg) & $74,60 \pm 14,00$ & $51,10 \pm 1,30$ & $68,96 \pm 15,39$ \\
GEO & $1,017 \pm 0,004$ & $1,012 \pm 0,006$ & $1,015 \pm 0,005$ \\
FCmáx (lat/min) & $191,8 \pm 2,0$ & $193,5 \pm 0,4$ & $192,3 \pm 1,8$ \\
T (min) & $20,96 \pm 0,77$ & $21,06 \pm 0,58$ & $20,99 \pm 0,70$
\end{tabular}

Nota: GEO (gravedad específica de la orina); t (tiempo que estuvo colocado el parche en minutos).

Entre las condiciones $\mathrm{C}$ se encontraron diferencias estadísticamente significativas $\left(19,2 \pm 12,6 \mu \mathrm{L} * \mathrm{~min}^{-1}\right)$ y también en las $A\left(14,6 \pm 10,3 \mu \mathrm{L} * \mathrm{~min}^{-1}\right)(\mathrm{p}=0,001)$ en la tasa de sudoración de la zona escapular, es decir, los sujetos sudaban menos cuando se les aplicaba antitranspirante comparado con la piel sin antitranspirante (ver figura 2).

\section{DISCUSIÓN}

El hallazgo más importante de este estudio fue que el método de parches sí es capaz de detectar la disminución esperada en la tasa de sudoración, cuando se aplica un antitranspirante en la piel y se realiza ejercicio a intensidad moderada-alta en el calor durante 20 minutos. El antitranspirante inhibió la producción de sudor, lo cual disminuyó la transpiración en un 24 \% aproximadamente, en comparación con la zona control (sin antitranspirante). 


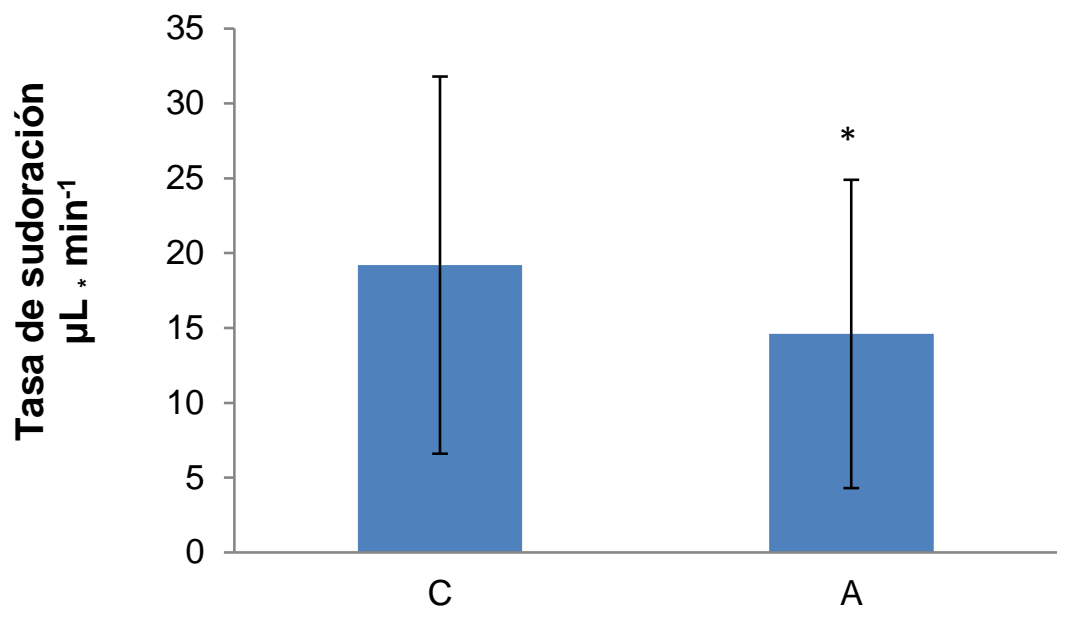

Figura 2. Resultado del ANOVA de una vía. C > A. Sin antitranspirante (C) $(19,2 \pm 12,6 \mu \mathrm{L}$ * $\min -1)$, con antitranspirante $(A)\left(14,6 \pm 10,3 \mu \mathrm{L}{ }^{*} \min -1\right) ;{ }^{*} p=0,001$

En un trabajo publicado por Burry, Evans, Rawling y Shiu (2003) estudiaron a dos grupos con 11 sujetos cada uno, los cuales debían ejercitarse durante 45 minutos en un cicloergómetro dentro de un cuarto de clima controlado $\left(35^{\circ} \mathrm{C}\right.$ y $20 \%$ de humedad relativa). En la primera sesión se le aplicaba desodorante después de 14 días de que el sujeto asegurara no haber utilizado algún producto antitranspirante bajo el brazo. La segunda sesión se realizó 7 días después de la primera y se utilizó un antitranspirante estándar del mercado. Los investigadores no encontraron diferencias significativas en cuanto a la tasa de sudoración (que se midió de manera general con el peso del sujeto antes y después del ejercicio) ni a la temperatura corporal entre el antitranspirante y el desodorante. En dicho estudio, los investigadores definieron la intensidad del ejercicio como aquella que cada participante pudo mantener en una prueba previa durante 45 minutos, sin exceder FCmax 10 pulsaciones por minuto, sin mencionar la composición de los productos utilizados y si los dejaron secar una vez aplicados sobre la piel, no controlaron el estado de hidratación de los sujetos (no lo midieron) además de no tener un grupo control y no aleatorizar.

El presente estudio, en cambio, se diseñó cuidadosamente para controlar debidamente la mayoría de las posibles fuentes de error: al realizar ambas mediciones al mismo tiempo y en la misma persona, la temperatura corporal y el estado de hidratación de cada participante fueron idénticas para ambas condiciones, como también lo fueron el tiempo de ejercicio y las condiciones ambientales. La aleatorización de la asignación del antitranspirante a un lado u otro, así como la evidencia previa que indica la ausencia de diferencias en la tasa de sudoración entre la zona escapular derecha y la izquierda (Havenith et al., 2008, Smith \& Havenith, 2011), completan la solidez del diseño. Finalmente, se reconoce que una debilidad del método presentado es el no describir cómo se puede aplicar una cantidad estandarizada de antitranspirante cada vez, lo cual sería indispensable para algunas investigaciones 
futuras. Sin embargo, esta prueba demuestra que sí es factible detectar el efecto esperado de un antitranspirante sobre la tasa de sudoración con la utilización del método de parches.

\section{REFERENCIAS}

Adams, R. (April, 1999). Revised physical activity readiness questionnaire. Canadian Family Physician, 45, 992. 995, 1004-1005. Recuperado de: http://www.ncbi.nlm.nih.gov/pmc/articles/PMC2328306/

Aragón-Vargas, L. F., Moncada-Jiménez, J., Hernández, J., Barrenechea, A., \& Monge-Alvarado, M. (2009). Evaluation of pre-game hydration status, heat stress, and fluid balance during professional soccer competition. European Journal of Sport Sciences, 9(5), 269-276. Recuperado de: http://dx.doi.org/10.1080/17461390902829242

Bain, A., Deren, T. M., \& Jay, O. (August, 2011). Describing individual variation in local sweating during exercise in a temperate environment. European Journal of Applied Physiology, 111(8), 1599-1607. Recuperado de: http://dx.doi.org/10.1007/s00421-010-1788-9

Burry, J., Evans, R., Rawlings, A., \& Shiu, J. (August, 2003). Effect of antiperspirants on whole body sweat rate and thermoregulation. International Journal of Cosmetic Science, 25(4), 189-192. Recuperado de: http://dx.doi.org/10.1046/j.1467-2494.2003.00184.x

Burton, A. C. (May, 1934). A new technic for the measurement of average skin temperature over surfaces of the body and the changes of skin temperature during exercise. The Journal of Nutrition, 7(5), 481-496. Recuperado de: http://jn.nutrition.org/content/7/5/481.short

Gellis, R., Goslin, B., Olson, R., McDonald, A., Russi, G., \& Moudgil, V. (May, 2007). Longitudinal modeling on the relationship between age and maximal heart rate. Medicine and Science in Sport and Exercise, 39(5), 822-829. Recuperado de: http://dx.doi.org/10.1097/mss.0b013e31803349c6

Havenith, G., Fogarty, A., Bartlett, R., Smith, C., \& Ventenat, V. (2008). Male and female upper body sweat distribution during running measured with technical absorbents. European Journal of Applied Physiology, 104(2), 245-255. Recuperado de: http://dx.doi.org/10.1007/s00421-007-0636-z

International Society for the Advancement of Kinanthropometry. (2001). International standards for anthropometric assessment. Australia: International Society for the Advancement of Kinanthropometry. Recuperado de: http://www.ceap.br/material/MAT17032011184632.pdf

Maughan, R. J., Merson, S. J., Broad, N. P., \& Shirreffs, S. M. (June, 2004). Fluid and electrolyte intake and loss in elite soccer players during training. International Journal of Sport Nutrition and Exercise Metabolism, 14(3), 327-340. Recuperado de: 
http://journals.humankinetics.com/ijsnem-back-

issues/IJSNEMVolume14Issue3June/FluidandElectrolytelntakeandLossinEliteSoccerPlay ersDuringTraining

Morris, N. B., Cramer, M. N., Hodder, S. G., Havenith, G., \& Jay, O. (2013). A comparison between the technical absorbent and ventilated capsule methods for measuring local sweat rate. Journal of Applied Physiology, 114(6), 816-823. Recuperado de: http://dx.doi.org/10.1152/japplphysiol.01088.2012

Oppliger, R., \& Bartok, C. (December, 2002). Hydration Testing of Athletes. Sports Medicine, 32(15), 959-971. Recuperado de: http://dx.doi.org/10.2165/00007256-200232150-00001

Phillips, M., \& McAllon, M. (October, 2008). A sweat-patch test for alcohol consumption: Evaluation in continuous and episodic drinkers. Alcoholism: Clinical \& Experimental Research, 4(4), 391-395. Recuperado de: http://dx.doi.org/10.1111/j.15300277.1980.tb04837.x

Reynolds, K., Darrigrand, A., Roberts, D., Knapik, J., Pollard, J., Duplantis, K., \& Jones, B. (October, 1995). Effects of antiperspirant with emollients on foot-sweat accumulation and blister formation while walking in the heat. Journal of the American Academy of Dermatology, 33(4), 626-630. Recuperado de: http://dx.doi.org/10.1016/0190$\underline{9622(95) 91283-5}$

Schmidt-Rose, T., Lehmbeck, F., Bürger, A., Windisch, B., Keyhani, R., \& Max, H. (2013). Efficient sweat reduction of three different antiperspirant application forms during stressinduced sweating. International Journal of Cosmetic Science, 35, 622-631. Recuperado de: http://dx.doi.org/10.1111/ics.12086

Shirreffs, S., Aragón-Vargas, L., Chamorro, M., Maughan, R., Serratosa, L., \& Zachwieja, J. (2005). The Sweating Response of Elite Professional Soccer Players to Training in the Heat. International Journal of Sports Medicine, 26(2), 90-95. Recuperado de: http://dx.doi.org/10.1055/s-2004-821112

Smith, C., \& Havenith, G. (July, 2011). Body mapping of sweating patterns in male athletes in mild exercise-induced hyperthermia. European Journal of Applied Physiology, 111(7), 13911404. Recuperado de: http://dx.doi.org/10.1007/s00421-010-1744-8

Participación: A- Financiamento, B- Diseño del estudio, C- Recolección de datos, D- Análisis estadístic e interpretación de resultados, E- Preparación de manuscrito. 